\title{
Students' Clinical Learning in an Emerging Dental School: An Investigation in International Collaboration Between Michigan and Ghana
}

\author{
Mathilde C. Peters, D.M.D., Ph.D., FADM, FICD; Francis Adu-Ababio, B.D.S., \\ M.Sc., DDPHRCS, FDSRCS, FWACS, FGCS; Nejay P. Jarrett-Ananaba, D.D.S.; \\ Lynn A. Johnson, Ph.D. \\ Abstract: The dearth of dental faculty members is a widely known problem that is exacerbated in countries that are attempting to \\ begin dental education programs. This collaboration between Kwame Nkrumah University of Science and Technology and the \\ University of Michigan investigated if dental students who have just started their clinical dental education can learn the knowl- \\ edge and skills required for identifying and restoring cavitated caries lesions through compact course delivery. There were three \\ instructional blocks: 1) didactic seminar; 2) seminar, simulated hands-on skills instruction, and clinical observation/assisting with \\ treatment of schoolchildren; and 3) seminar, simulated skills training, and application to schoolchildren. Each dental student com- \\ pleted a questionnaire measuring knowledge and perceptions of knowledge, experience, and confidence at five points in time. The \\ dental students' knowledge increased significantly as well as their perceived knowledge, experience, and confidence ( $p<0.0001)$. \\ In general, the students showed proficiency in delivering simple treatments. The project showed that an integrated compact \\ course delivery model may assist emerging dental schools to cope with the challenging shortage of resident faculty members.
}

Dr. Peters is Professor of Dentistry, Department of Cariology, Restorative Sciences, and Endodontics, School of Dentistry, University of Michigan; Dr. Adu-Ababio is Dean, Dental School, College of Health Sciences, Kwame Nkrumah University of Science and Technology, Kumasi, Ghana; Dr. Jarret-Ananaba is Clinical Lecturer, Department of Periodontics and Oral Medicine, School of Dentistry, University of Michigan; and Dr. Johnson is Professor of Dentistry and Assistant Dean for Informatics and Innovation, Department of Periodontics and Oral Medicine, School of Dentistry, University of Michigan. Direct correspondence and requests for reprints to Dr. Mathilde Peters, School of Dentistry, University of Michigan, D-2391, 1011 N. University, Ann Arbor, MI 48109; 734-763-3366; mcpete@umich.edu.

Keywords: international cooperation, dental caries, dental education, clinical education, Ghana

Submitted for publication 12/23/11; accepted 12/9/12

$\mathrm{O}$ ne of the challenges newly established health professions education programs encounter is how to attract and retain resident faculty members to develop and deliver a sustainable curriculum. The fast pace with which new dental schools have been opening in many countries (e.g., United States, Australia, Brazil) alerts dental educators to a global problem. ${ }^{1}$ The current shortage of dental faculty members in general only aggravates the problem. To assist in the initial phases of establishing educational programs, new schools draw on experienced faculty members from existing institutions. ${ }^{2}$ While the faculty workforce shortage is well documented in dental education, ${ }^{3,4}$ the problem is compounded in developing countries. When establishing or expanding their health care systems, these countries are faced with a lack of trained local workforce. ${ }^{5}$ Commonly, health care workers would receive advanced training in a foreign country. ${ }^{5}$ However, after completing their training, few return to improve the health of the people in their home country. ${ }^{6}$

To assist with this problem, in 1989, the University of Michigan (U-M) collaborated on the first in-country postgraduate $\mathrm{OB} / \mathrm{GYN}$ training program in Ghana. Since then, 95 percent of its graduates have stayed in Ghana. ${ }^{7}$ Building on that success, the U-M committed in 2008 to expand its health care collaboration with Ghana. Thus, the dental schools at two universities-Kwame Nkrumah University of Science and Technology (KNUST) in Ghana and the $\mathrm{U}-\mathrm{M}$ - have formed a mutually beneficial research and educational collaboration. ${ }^{8}$ This collaboration 
resulted in grant support from the Bill and Melinda Gates Foundation to develop and evaluate pilot projects that would improve and support health education and research in Ghana.

This pilot project investigated if compact course delivery through faculty exchange can have an impact on a developing educational environment. ${ }^{2}$ This article describes the first collaborative educational research project: its aim was to explore if dental students who have just started their clinical dental education can, through compact course delivery, learn the knowledge and skills required for identifying and restoring cavitated caries lesions using a simple technique. ${ }^{9}$

\section{Oral Health Needs and Education in Ghana}

A growing body of evidence supports the impact of oral health on general health and emphasizes the often two-way existing link between oral health and systemic diseases such as diabetes and heart disease. ${ }^{10}$ Caries infection is one of the most common childhood diseases. ${ }^{11}$ Caries prevalence in Ghanaian children is moderately high in urban communities and is increasing at an alarming rate. ${ }^{12}$ It is important to intercept the caries infection early on. In addition to the suffering that comes with caries disease and its sequelae, children with carious dentitions perform poorly in school, and their social well-being is greatly hampered. Delivery of sustainable oral health care by control and prevention of local oral infections in children will promote their lifelong oral and general health.

Ghana has an estimated population of 23.42 million people..$^{13}$ There is a tremendous need for oral health care and oral health providers in Ghana. The country has approximately 150 dentists, 70 percent of whom are based in Accra and Kumasi. ${ }^{14}$ Dental training started at the University of Ghana Medical School at Korle-Bu, Accra, Ghana, in 1974. After two years of local training, dental students were government-sponsored to continue their training in Lagos, Nigeria, or Manchester, UK. However, training in Nigeria was discontinued, and the dental students were sent to King's College, London, while the Manchester arrangement remained. In 1992, the entire training in dentistry was localized at the country's first dental school, University of Ghana Dental School in Accra, and the first cohort of five dental surgeons fully trained at this Ghanaian dental school graduated in 1997. In 2006, the second dental school was established as the Kwame Nkrumah University of Science and Technology (KNUST) Dental School, located in Kumasi, Ghana. In August 2012, the KNUST Dental School graduated its first cohort of dental surgeons.

Dental students at KNUST first enter into the three-year Bachelor of Science in Human Biology program. At this stage of their training, students follow the same curriculum as their medical cohorts. On completion of the human biology program, the dental students are awarded the B.Sc. (Human Biology) degree, before proceeding to do their three-year clinical training in dentistry, which leads to the Bachelor of Dental Surgery (B.D.S.) degree.

The purpose of this project was to investigate the ability of a compact educational program to assist in the learning process of novice Ghanaian dental students. The program aimed to prepare first-year clinical dental students for delivery of simple, practical oral health care. Targeted educational interventions included evidence-based learning and practical instruction for skills development, followed by an applied clinical component that aimed to facilitate experiential learning. ${ }^{15}$ The related clinical component investigated if these dental students at the start of their dental curriculum were capable of making a contribution towards improving the oral health of school children. The effect of the educational intervention was determined using surveys on student perception and student-learning efficacy.

\section{Methods}

The inaugural class (eight first-year students) of the newly established dental school in Kumasi, Ghana, participated in this project. A short course, Applied Cariology: Healthy Teeth, was developed to educate and train the novice dental students in delivery of sustainable oral health care by means of Atraumatic Restorative Treatment (ART). ${ }^{16}$ The educational intervention was initiated at the end of a three-year general medical training program, prior to the students starting their three-year dental education. The program was conducted in three blocks (in total eight teaching days) over a period of eight months. Two resident dentists and two experienced community oral health officers (mid-level dental professionals) participated as adjunct teachers. Twenty-eight schoolchildren (six to nine years old) from two primary schools in Kumasi, Ghana, with 
active caries disease participated in the applied part of the project. The educational and clinical intervention was approved by the governing Institutional Review Boards at the universities of Michigan and Kwame Nkrumah.

The course is centered around sustainable, minimally invasive management of caries disease. The content was targeted for students with general medical background who had not yet started dental training. The program was delivered in three blocks (A, B, and C), spaced in time (Figure 1). After introduction to basic information of the oral environment, its structures, and tissues, the program focused on the caries disease process. Basic information of the multifactorial nature of the disease, factors involved in the initial disease process, the dynamics of disease progression, and its effect on the hard tooth tissues were taught in interactive seminar format. Subsequently, with a focus on minimally invasive caries management, basic caries removal and simple restorative care (ART) were introduced as a sustainable technique to manage cavitated lesions. Two threehour seminars (distributed over two blocks) were followed by hands-on training and skills development in a laboratory setting. Students were then provided with their first clinical experiences: observing and assisting with ART treatments under field conditions. In a third block, evidence-based learning experiences and skills training were continued, leading to their first clinical treatment experience providing prevention and ART treatment to school children.

Starting in Block A, a three-hour seminar familiarized the students with the basics of cariology (Figure 1). In Block B, a second three-hour seminar was followed by hands-on skills development (ART) and clinical experience (observe/assist) in ART treatment under field conditions (Table 1). Block C consisted of further evidence-based learning experiences and skills training. This block captured and grounded all learning experiences up to that point, leading up to students' first clinical experience of treating the participating school children. To follow their knowledge acquisition and clinical learning process, the students were surveyed and tested at five points in time (Figure 1). Concurrently, the project demonstrated clinical care delivery in a team setting by community oral health officers and dentists/dental students collaborating together.

The researchers employed a pre-post questionnaire time series design to investigate the students' learning and their perceptions of their knowledge, experience, and confidence. The small class size of eight students did not allow for a control group. All eight dental students took the same questionnaire five times. Between each questionnaire, there was either a time gap of no activity or an educational intervention. While correlated data from repeated measures on the same subjects in a longitudinal study are a threat to

\section{Time line}

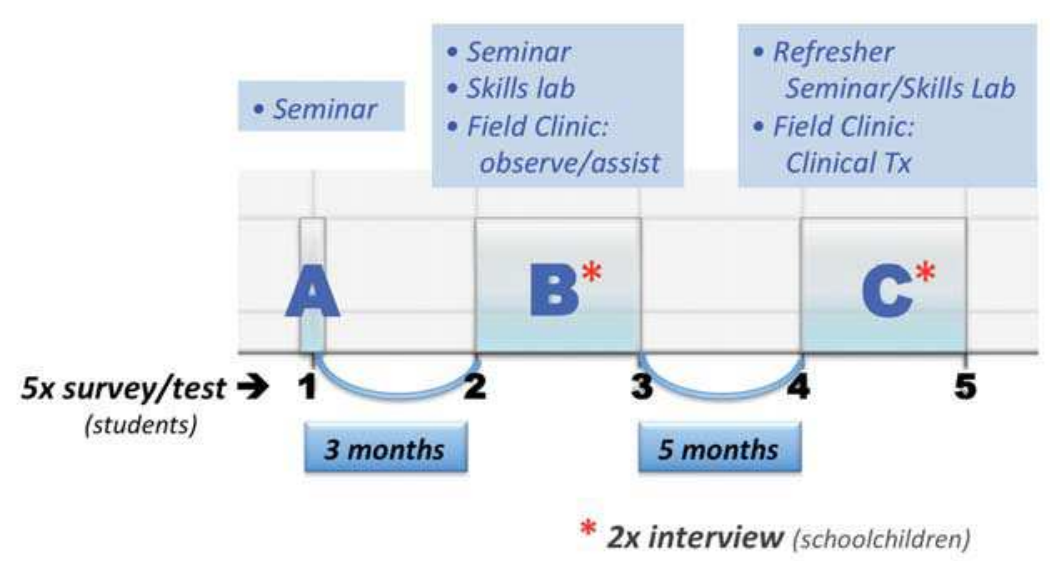

Figure 1. Time line of educational experiences offered indicating educational Blocks A, B, and C, times of PPI-student surveys (1-5), and children's interviews $\left(2 x^{*}\right)$ 
Table 1. Student learning: didactic and clinical learning activities during Blocks A, B, and C

\begin{tabular}{lcc} 
Period & Didactic/Clinical Learning & Number of Hours \\
\hline Block A & Seminar & $3 \mathrm{hrs}$ \\
Block B & Seminar & $3 \mathrm{hrs}$ \\
& Skills lab & $2 \times 2 \mathrm{hrs}$ \\
Block C & Field clinic: observe/assist & $2 \times 4 \mathrm{hrs}$ \\
& Refresher seminar & $1 \mathrm{hr}$ \\
& Refresher skills lab & $2 \mathrm{hrs}$ \\
& Field clinic: clinical treatments & $2 \times 5 \mathrm{hrs}$
\end{tabular}

internal validity, the use of the Participant Perception Indicator is an established research approach. ${ }^{17,18}$

Traditional seminars with interactive questionand-answer time were used to teach the basic concepts of cariology (Blocks A, B, and C). Hands-on instruction in a mannequin-based simulation facility was used to teach basic manual skills including ART to the students (Block B). This was followed by two days of clinical field experiences. During the first field clinic (Block B), the treatments were provided by two resident dentists and two community oral health officers (mid-level dental professionals) in an oral health care team setting. This allowed for ample clinical observe-and-assist opportunities for the students. During the second field clinic (Block C), the students provided patient care under close oversight by licensed dentists and community oral health officers.

At the five points described, a two-part dental student questionnaire was administered to the students in order to measure their perceptions of their own knowledge, experience, and confidence to treat patients with simple carious lesions as well as their actual knowledge of the caries disease process. The first part was the Participant Perception Indicator (PPI), ${ }^{17}$ an assessment instrument that measures perceptions of themes determined important for learning. ${ }^{18,19}$ This instrument measured students' perception of three themes: their knowledge, experience, and confidence in caring for children with caries-affected teeth. It was administered five times to measure the change in students' perceptions over the course of the project. For each theme, multiple statements were used to increase reliability. The PPI questionnaire can be found at open.michigan (http:// open.umich.edu/). Directions were as follows: "The following questions are designed to measure your perception of your knowledge, experience, and confidence on various aspects of cariology and patient care. For each question, indicate how you feel about your knowledge, experience, and confidence."

The second part of the questionnaire was for knowledge assessment. The knowledge test was comprised of a series of true/false and short-answer questions that measured the students' knowledge of the caries disease process and its treatment in children. It was given five times to measure the changes in the students' knowledge during the project. The knowledge questionnaire and answer key can be found at open.michigan (http://open.umich.edu/). At the start of the project, the residents and dental auxiliaries were tested to determine a gold standard.

Student data from the PPI and knowledge test were analyzed with mixed models with repeated measures (significant at 0.05 level) using SPSS for Windows, version 19.0.0 (SPSS Inc., Chicago, IL, USA). The mixed-effects models procedure handles correlated data and unequal variances..$^{20,21}$ Correlated data are very common in such situations as repeated measurements of survey respondents. The mixedeffects models procedure extends repeated measures models to allow an unequal number of repetitions. In a mixed-effects model, responses from a subject are thought to be the sum of so-called fixed effects (affecting the population mean) and random effects (associated with a sampling procedure such as subject effect). The mixed-effects model procedure solves these problems by estimating fixed and random effects in one model.

\section{Results}

For each of the three themes (knowledge, experience, and confidence), the students perceived a significant increase between start of the course and its conclusion (Table 2: interval 1 vs. $5, \mathrm{p}<0.0001$ ). For the PPI theme on knowledge, the variation in knowledge among students greatly converged over 
Table 2. Significance of pairwise comparison between intervals based on estimated marginal means

\begin{tabular}{lccc} 
Intervals & Knowledge & Experience & Confidence \\
\hline 1 vs. 2 & $<0.0001$ & 0.034 & 0.014 \\
1 vs. 3 & $<0.0001$ & 0.001 & $<0.0001$ \\
1 vs. 4 & $<0.0001$ & $<0.0001$ & $<0.0001$ \\
1 vs. 5 & $<0.0001$ & $<0.0001$ & $<0.0001$ \\
2 vs. 3 & 0.026 & 0.109 & 0.069 \\
2 vs. 4 & $<0.0001$ & $<0.0001$ & $<0.0001$ \\
2 vs. 5 & $<0.0001$ & $<0.0001$ & $<0.0001$ \\
3 vs. 4 & 0.019 & 0.001 & 0.002 \\
3 vs. 5 & 0.013 & 0.000 & $<0.0001$ \\
4 vs. 5 & 0.877 & 0.310 & 0.404 \\
Note: Mean difference is significant at 0.05 level. & &
\end{tabular}

time, ranging from 10 to 24 out of a possible 35 score at entrance level and to 31 to 35 at exit level (Figure 2). Their perceived knowledge increased after Block A (interval 1 to $2, \mathrm{p}<0.0001$ ) and increased again after Block B (interval 2 to $3, p=0.026$ ). Their knowledge continued to increase after theoretical and skills refresher (interval 3 to $4, p=0.019$ ). Their final clinical field experience in Block $C$ then supported and sustained their high knowledge level (not significant): interval 4 to $5, \mathrm{p}=0.877$.

For the PPI theme on experience, the students' perception showed a significant increase in experience (interval 1 to $2, p \leq 0.034$; Figure 3 ). Their perceived experience significantly increased between Blocks B and C (interval 3 to $4, p=0.001$ ) and was then sustained (interval 4 to $5, \mathrm{p}=0.310$ ). This period included a limited general exposure to the dental field (theoretical courses and limited preclinical laboratory exercises). One student showed a very modest selfrating of his or her own experience.

For the PPI theme on confidence, students' perception of their confidence showed a significant increase after Block A (interval 1 to 2, $\mathrm{p} \leq 0.014$; Figure 4). This confidence consolidated after skills training in Block B (interval 2 to $3, p<0.069$ ) and reached a significant higher level after the theoretical and skills refresher at the start of Block $\mathrm{C}$ (interval 3 to $4, \mathrm{p}<0.002$ ). The students' confidence was sustained during their own clinical treatment phase in Block C (interval 4 to $5, \mathrm{p}=0.404$ ).

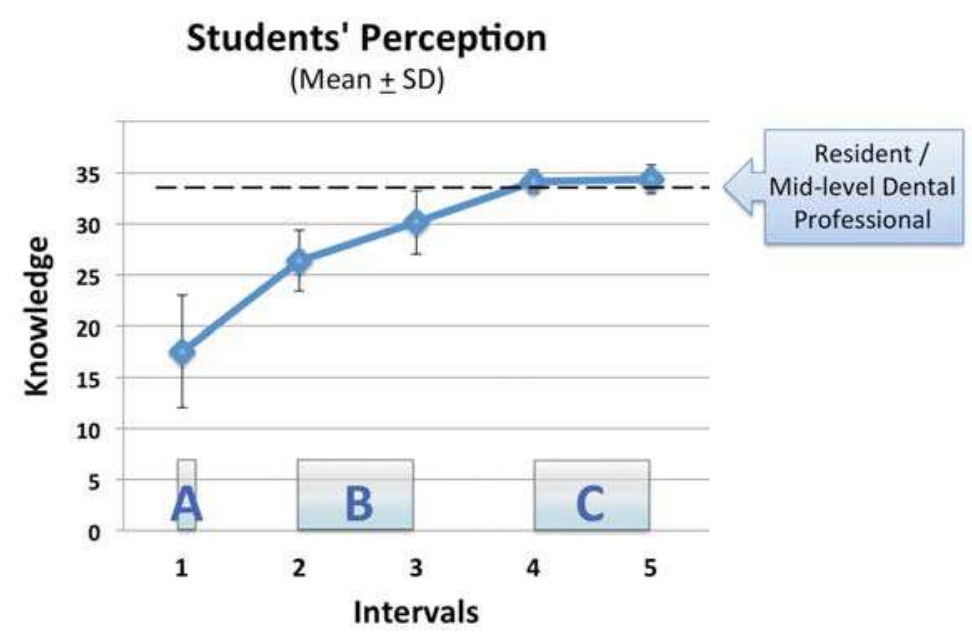

Figure 2. Students' perception of their level of knowledge in course periods A-C

Note: Significant increases were found between intervals 1 and 2, 2 and 3, and 3 and 4. Between 4 and 5, no difference was noted. 


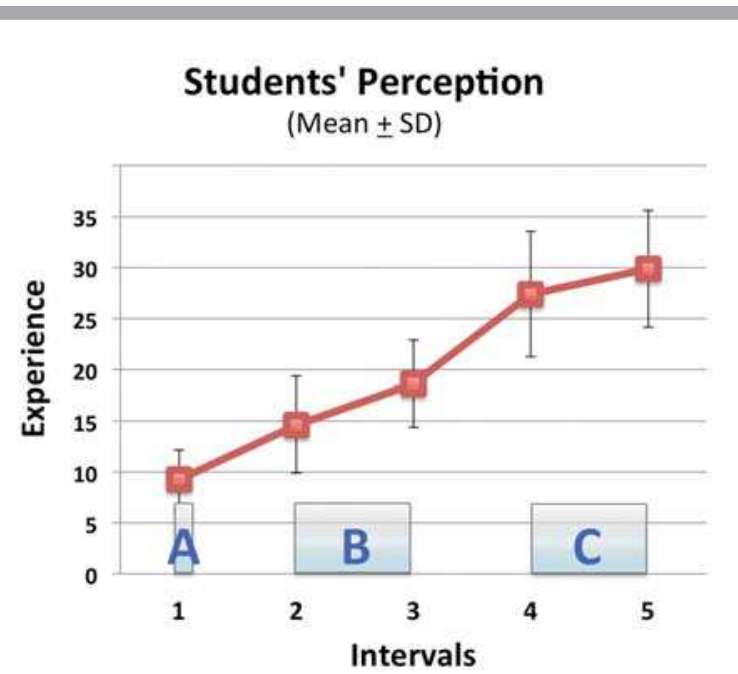

Figure 3. Students' perception of their level of experience in course periods A-C

Note: Significant increases were found between intervals 1 and 2 and between 3 and 4 . Between 2 and 3 and between 4 and 5, little difference was noted.

The knowledge test provided a comparative measure against the knowledge perception outcomes (Figure 5). Significant differences between perception (PPI-K) and knowledge tests were found for open questions at interval $1(\mathrm{p}=0.021)$ and for both open and T/F questions at intervals 4 and $5(\mathrm{p} \leq 0.005)$.

\section{Students' Perception}

(Mean \pm SD)

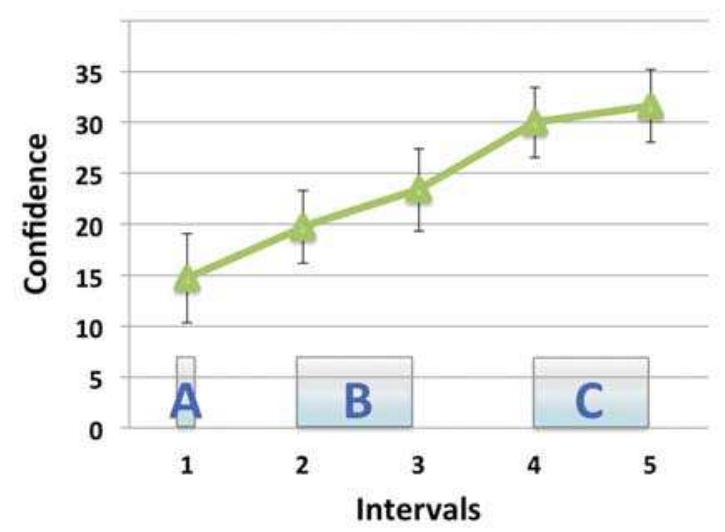

Figure 4. Students' perception of their level of confidence in course periods A-C

Note: Significant increases were found between intervals 1 and 2 and between 3 and 4 . Between 2 and 3 and between 4 and 5, little difference was noted.

\section{Limitations}

This project was the first instructional research study as well as the first clinical research study that Kwame Nkrumah University of Science and Tech-

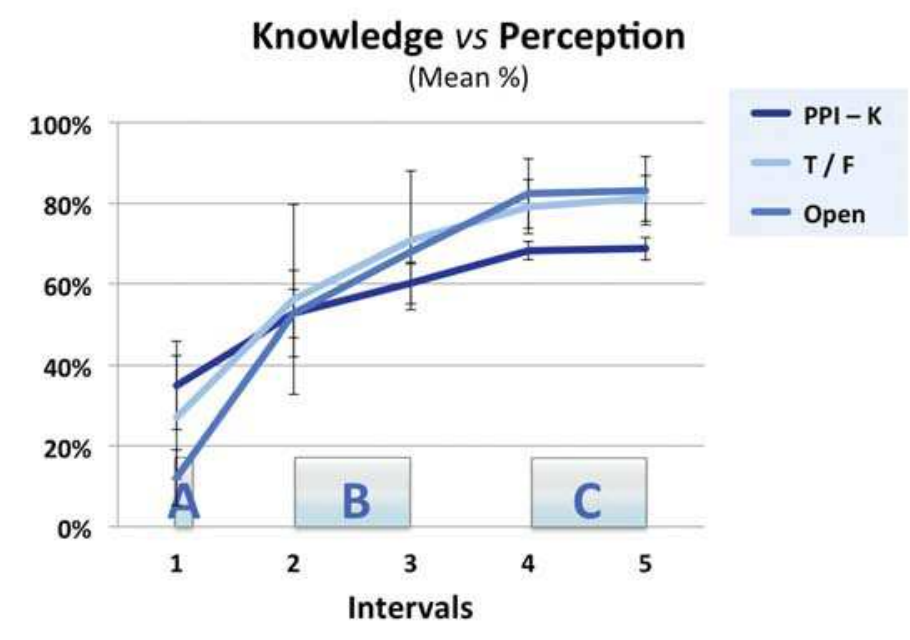

Figure 5. Students' perception of their knowledge (PPI-K) and test knowledge (open questions and true/false questions) in course periods A-C

Note: Significant differences between perception (PPI-K) and tests were found for open questions at intervals 1, 4, and 5 and for true/ false questions at intervals 4 and 5 . 
nology (KNUST) Dental School had been involved in. As a new school that graduated its first class in August 2012, KNUST has been focusing on establishing itself as Ghana's second dental school before launching a research program. The limitations of conducting instructional research in this setting were many. For the instructional research component, two major limitations existed: a small sample size and poor external validity. The project was conducted with the inaugural class of eight students. This small size prevented the use of a control group as well as random assignment to a group. There are threats to validity of the findings by using a pre- and posttest design when no control group is available. The data observed might be a function of multiple exposures to the questionnaire as subjects may learn from their experiences, providing correlated data that poses a threat to internal validity. The same questionnaire was repeated five times over the course of one year. Consequently, the students could have learned information from sources external to the research project. By design, posttest differences might have been attributable to characteristic differences between subjects rather than to the intervention.

The struggle of starting a dental school in an emerging country means that faculty members are brought in for short periods of time from across the world to teach in their area of expertise. The curricular controls that we take for granted in established academic dental institutions will eventually be in place, but at the time of this research they were not yet mature. Thus, small group size and significant threats to external validity mean that the study was limited to observing the single group and the findings cannot be generalized to a larger student population.

\section{Discussion}

The dental student questionnaire consisted of a knowledge test and a PPI that measured the dental students' perception of their knowledge, experience, and confidence at the beginning, middle, and end of the study. The knowledge test showed that the students' knowledge significantly increased over time $(p<0.003)$. This increase happened faster and was larger than the students perceived (Figure 5). The perceptions of all themes increased significantly $(p<0.0001)$ with the perceptions of knowledge increasing the most $(p<0.0001)$ during the first half of the course (Figures 2-4).

Over the course of eight months, dental students new to patient care studied the caries process and how to care for children with cavitated carious lesions using ART. The students' knowledge of the caries disease process increased as well as their perceptions of their knowledge, experience, and confidence in caring for children with dental caries. The students' perception of their knowledge, as measured by the PPI, reached a proficiency level similar to resident dentists and community oral health care workers and sustained that level to the end of the study. Their experience and confidence continued to increase to the end of the study and was then sustained.

The short course was designed to offer students learning opportunities and engage them in the subject during three brief immersion periods. During the time intervals between Block A and Block B and again between Block B and Block C, the students continued with their regular dental curriculum. It is anticipated that their dental studies combined with the instruction of this project would increase the students' knowledge of dental topics and patient care in general. As their actual knowledge increased, it is interesting to note that their perception of their knowledge decreased (Figure 5). This might have been due to the adage that the more you know, the more you realize how much more there is to learn. At the start of the course, the students' perception of their knowledge was higher than the actual knowledge test responses indicated (significantly different for open questions). At the start of Block B, however, this difference had disappeared, and perception was in line with their knowledge. From then on, their true knowledge as measured ( $\mathrm{T} / \mathrm{F}$ and open questions) was actually better than their own perception of their knowledge would predict. Comparison for Block $\mathrm{C}$ resulted in significantly higher $(\mathrm{p} \leq 0.005)$ knowledge levels at intervals 4 and 5 (Figure 5). The interventions provided at three- and five-month intervals without any course-specific educational maintenance within these intervals appeared to have been effective.

Because none of the dental students had previous experience in patient care, no benchmark was determined at the start of the course. The spread in starting level may reflect the students' uncertainty with respect to self-rating of experience. The variation among student responses was further increased at the start of Block B.

The applied clinical component was designed to facilitate and impact the dental students' learning by giving them an opportunity to learn from personal experience what providing oral health care can mean to a group of children and how their own learning immediately allows them to contribute to improve 
oral health. The presence of the stages of experience (doing), reflection, and application or conceptualization appeared to make the experiential learning different and powerful. ${ }^{15}$

Interestingly, two teeth that had lost their restorations between Block B and Block C but were not in need of a replacement restoration provided occasion for a thorough discussion of the caries process. The previously soft carious dentin, left behind in the central part of the cavity, was now hard and appeared to be remineralized upon probing with an explorer (no tug-back). The caries process was halted, eliminating the need for any further treatment. Observing the differences in active and arrested caries in this group of children provoked extensive discussions about biological remineralization and stabilization of lesions. This phenomenon, clinically apparent in two very different cavities, provided excellent clinical learning moments for the students. The project showcased an interactive learning model that combined knowledge building and skills development with applied clinical experiences. The students transitioned from being not knowledgeable about ART and having no practical skills to being proficient in delivering sustainable oral health care to children. Each dental student had performed multiple ART procedures at the end of the study. The clinical experience integrated all aspects of the course and had a motivating effect on the students. With limited expertise and time, they were able to contribute to the children's oral health.

The project showed that an integrated compact course delivery model may assist emerging dental schools to cope with the challenging shortage of resident faculty members. Planned expansion of the student population warrants future instructional research including a student control group.

\section{Conclusion}

The targeted, compact course Applied Cariology: Healthy Teeth including limited guided clinical experiences provided a learning experience that proved to be successful in transitioning the novice dental students into dental care providers who can have an impact on children's oral health and wellbeing. The limited, simple ART field treatments showcased in this course demonstrated that novice dental students can relieve and reduce clinical dental problems in children. The strategy of didactic instruction followed by relevant hand skills training in a simulation setting, observation and assisting of experienced clinicians in a clinical setting, and concluding with actual clinical experiences with children proved to be a feasible alternative to conventional classroom instruction. The course functioned as a motivator for students to become dentist-providers in a professional oral health care team.

This collaborative research study demonstrated that novice dental students quickly learn clinical skills and are able to apply them to patients in their community. The instruction, however, needs to be carefully planned to combine locally feasible didactic and hands-on skills training and involve applied patient care. Connecting instruction and patient care elements in a team approach early on in the educational process concurrently allowed the students to enhance children's oral health.

\section{Acknowledgments}

The authors are grateful for the support of the Bill and Melinda Gates Foundation. Instruments and materials for preclinical and clinical patient care were generously provided by GC America Inc (Alsip, IL, USA) and Colgate-Palmolive Company (New York, NY, USA). We thank the team of resident dentists and community oral health officers and the entire staff of KNUST Dental School for their assistance with this project. The enthusiasm of teachers and students of the two primary schools made this collaborative project a success.

\section{REFERENCES}

1. Reynolds PA, Eaton KA, Paganelli C, Shanley D. Nine years of DentEd: a global perspective on dental education. Br Dent J 2008;205(4):199-204.

2. Allen DL, Puente ES. The involvement of United States dental schools in international dental education. J Dent Educ 1989;53(10):593-4.

3. John V, Papageorge M, Jahangiri L, et al. Recruitment, development, and retention of dental faculty in a changing environment. J Dent Educ 2011;75(1):82-9.

4. Corbet E, Akinwade J, Duggal R, et al. Staff recruitment, development, and global mobility. Eur J Dent Educ 2008;12(Suppl 1):149-60.

5. Jeboda SO. Dental education in Africa, with special reference to Nigeria. Int Dent J 1997;47(1):21-5.

6. White JG. Interacting forces influencing private dental practice in South Africa: implications for dental education. S Afr Dent J 2008;63(2):80-5.

7. Johnson J. Dr. Tim Johnson gets hooked on Ghana. University of Michigan Center for Global Health Newsletter, December 11, 2011. 
8. Bill and Melinda Gates Foundation Planning Grant. The Ghana-Michigan collaborative health alliance for reshaping training, education, and research (CHARTER), \#085779, 2010.

9. Lalumandier JA, Victoroff KZ, Thuernagle O. Early clinical experience for first-year dental students. J Dent Educ 2004;68(10):1090-5.

10. Li X, Kolltveit KM, Tronstad L, Olsen I. Systemic diseases caused by oral infection. Clin Microbiol Rev 2000;13(4):547-58.

11. Moynihan PJ. The role of diet and nutrition in the etiology and prevention of oral diseases. Bull World Health Organ 2005;83(9):694-9.

12. Bruce I, Addo ME, Ndanu T. Oral health status of peri-urban school children in Accra, Ghana. Int Dent $\mathrm{J}$ 2002;52(4):278-82.

13. Ghana Statistical Service. Ghana housing and population census, 2009. At: www.statsghana.gov.gh/. Accessed: November 22, 2011.

14. Ofori MA, Adu-Ababio F, Nyako EA, Ndanu T. Prevalence of dental fear and anxiety amongst patients in selected dental clinics in Ghana. Health Educ J 2009;68(2):130-9.
15. Proudman B. Experiential education as emotionally engaged learning. In: Warren K, Sakofs M, Hunt JS Jr, eds. The theory of experiential education. Dubuque, IA: Kendall/Hunt Publishing, 1995.

16. Frencken JE, Pilot T, Songpaisan Y, Phantumvanit P. Atraumatic restorative treatment (ART): rationale, technique, and development. J Public Health Dent 1996;56(Spec Iss 3):135-40.

17. Berger C. Participant Perception Indicator (PPI) evaluation/assessment tool. At: http://sitemaker.umich.edu/ppi. evaluation.tool/home. Accessed: November 18, 2011.

18. Berger C, Barritt M. Evaluation of the location independent learning and teaching course: economics of networks. Ann Arbor: University of Michigan, 1998.

19. Berger C, Kerner N, Lee Y. Understanding student perceptions of collaboration, laboratory, and inquiry use in introductory chemistry. Boston: Association for Research in Science Teaching, 1999.

20. McCulloch CE, Searle SS. Generalized, linear, and mixed models. New York: Wiley and Sons, 2001.

21. Verbeke G, Molenberghs G. Linear mixed models for longitudinal data. New York: Springer, 2000. 